\title{
Análisis de las dificultades en la comprensión y aplicación del principio alfabético
}

\author{
José Escoriza Nieto
}

Departamento de Psicología Evolutiva y de la Educación, Univ. de Barcelona

España

j.escoriza@ub.edu 


\section{Resumen}

El objetivo de este trabajo, ha sido el de analizar la problemática conceptual general existente con respecto a la explicación de las dificultades específicas de aprendizaje del lenguaje escrito. Aunque algunos autores consideran que se ha alcanzado un cierto nivel de consenso, en algunas cuestiones puntuales, las discrepancias tan profundas, que siguen subsistiendo, con respecto a la conceptuación de las dificultades de aprendizaje y al proceso de lectura, nos anuncian un clima de controversia con futuro y al que, solamente, la investigación y la reflexión crítica pueden poner freno o, en su caso, atenuar. La preocupación básica, que se deriva del panorama actual acerca de la dislexia, no es solamente de tipo explicativo, sino que se hace extensivo, lógicamente, a los procedimientos que se siguen para evaluar la gravedad del problema y para ayudar a los alumnos/as, con dificultades de aprendizaje, a promover la internalización del lenguaje escrito como instrumento psicológico.

Palabras Clave: dislexia, principio alfabético, conocimiento fonológico, lenguaje escrito.

\section{Introducción}

La polémica, en torno a la explicación de las dificultades específicas en el proceso de aprendizaje del LE, tiene una larga tradición y ha seguido un proceso paralelo a la controversia generada en el ámbito de las Dificultades de Aprendizaje (DA). Esta problemática conceptual la podemos articular en torno a dos grandes perspectivas: la neuropsicológica y la defectológica o sociocultural (Escoriza, 1998ab, 2002). La adopción como referente de uno u otro marco teórico tendrá implicaciones determinantes tanto en lo que hace referencia a la interpretación de la dislexia como en lo relativo a la formulación de criterios para su evaluación y tratamiento educativo (Escoriza, 1998ab, Escoriza y Boj, 1998) (ver tabla 1). La supuesta implicación de las disfunciones neurológicas, en la explicación de la dislexia, ha dado lugar a la formulación de diversas líneas de investigación al entender que la dislexia es una manifestación diagnosticable de retraso en el aprendizaje del lenguaje escrito debido a la existencia de determinadas disfunciones de naturaleza orgánica identificadas en cualquiera de los siguientes sistemas: hemisferial, cerebelar, magnocelular y/o genético (Olson, 2002; Nicolson y Fawcett, 1999; Knight y Hynd., 2002; Eckert y Leonard, 2000; Frith, 1999, 2002; Pugh et al., 2000; Stein, 2001, entre otros). A los planteamientos, expuestos 
desde el modelo médico, le sucederían las explicaciones sustentadas por los defensores del modelo de los procesos cognitivos básicos (Escoriza, 1998b).

La tradición neuropsicológica se ha fundamentado en una serie de principios explicativos que han influido de forma notable en la interpretación de las dificultades específicas, en el aprendizaje del lenguaje escrito, que se han venido formulando:

a. El desarrollo como proceso interno: incremento cuantitativo de las funciones orgánicas y/o psicológicas cuya evolución normativa va a posibilitar el aprendizaje del lenguaje escrito $\mathrm{y}$, en consecuencia, la generación de cualquier tipo de disfunción va a ser entendida como la causante de la dislexia. Dichas disfunciones, constituyen obstáculos internos que limitan o dificultan el rendimiento y explican, por tanto, la discrepancia que pueda darse entre CI y aprendizaje del LE. Según Frith (1999), disfunción, déficit y anomalía, son todos ellos términos referidos al trastorno de una función normativa, causante de las dificultades para aprender a leer y escribir. Frith $(1999,2002)$ sostiene que está emergiendo un evidente consenso de que la dislexia es un trastorno neurológico con un origen biológico que influye en el procesamiento del lenguaje y con una amplia gama de manifestaciones clínicas. Esta autora (1999, págs. 197-198) propone emplear el término dislexia solamente cuando hagamos referencia a un trastorno neuroevolutivo y no para referirnos a problemas de lectura. En la cadena causal que formula, Frith entiende que si existe una disfunción a nivel neurológico, en un sistema específico del cerebro, entonces se producirá una disfunción en los procesos cognitivos, basados en dicho sistema, que serán los causantes de la dislexia. Toda este serie de causas distales (disfunciones neurológicas) y proximales (déficits cognitivos) tendrán una manifestación conductual clara y evidente: dificultades en el aprendizaje de la lectura. El criterio aplicado, para identificar a una persona como disléxica, es el criterio de discrepancia significativa.

b. A la hora de concretar qué tipo de poblaciones, pueden ser consideradas como no disléxicas, se aplica el criterio excluyente. La aplicación de este criterio suele tener dos versiones generales. Una de ellas, la encontramos en casi la totalidad de las definiciones de DA y la segunda la encontramos en la propuesta que formula Frith $(1999,2002)$ : el término disléxico queda reservado solamente a personas que presentan problemas de lectura debido a una disfunción neurológica y no cuando nos referimos a dificultades de lectura debidos a otro tipo de causas posibles. 
Análisis de las dificultades en la comprensión y aplicación del principio alfabético

c. Para la evaluación, de los efectos de la discapacidad, suelen emplearse procedimientos psicométricos: medición cuantitativa de aptitudes o funciones psicológicas. Según Kozulin (2000, p. 88), la evaluación, de acuerdo con el paradigma psicométrico, presenta las siguientes características: el nivel de rendimiento manifiesto, revela las aptitudes internas del niño con más o menos precisión, la ejecución sin ayuda, es el mejor formato para llevar a cabo la evaluación y el objetivo de las pruebas, es predecir el rendimiento futuro y clasificar al niño según su nivel de aptitud.

Cole y Griffin (1989, p. 89), han formulado la siguiente valoración de la tradición epistemológica que estamos analizando: "La adquisición de la lectura plantea en nuestras sociedades numerosos problemas que tradicionalmente se le han achacado al individuo como portador de déficits específicos que estarían en la base de cualquier dificultad a la hora de enfrentarse a un texto escrito". En el comentario general, que formulan Cole y Griffin, podemos resaltar tres elementos conceptuales: la existencia de una tradición epistemológica centrada en atribuir las dificultades de aprendizaje de la lectura y escritura a déficits internos (criterio intrínseco), la consideración del aprendizaje del lenguaje escrito como un proceso evolutivo, es decir, como un proceso en el que se va produciendo una internalización gradual de la competencia literada y la necesidad de que en este proceso se vayan sucediendo sistemas mediadores diferentes en función de las necesidades educativas identificadas.

La perspectiva sociocultural, por su parte, propone un marco explicativo radicalmente diferente al que hemos expuesto como propio de la tradición neuropsicológica:

a. El proceso de cambio evolutivo/educativo entendido como la interacción entre las líneas natural y cultural del desarrollo y las dificultades de aprendizaje atribuidas a factores de naturaleza sociocultural ya que, de acuerdo con Kozulin (2000), el principio constructor, de las funciones psicológicas superiores, se encuentra fuera del individuo: en los instrumentos psicológicos y en las relaciones interpersonales.

b. La discapacidad no tiene un efecto limitativo sobre el desarrollo, sino que puede generar un desarrollo diferente (Gindis, 1995). El que promueve modificaciones es el medio sociocultural y no la disfunción orgánica por sí misma. Kozulin (2000, p. 48), considera que para que tenga lugar una adecuada formación de conceptos, el niño debe participar en unas actividades de aprendizaje especialmente diseñadas que ofrezcan un marco de referencia para una construcción guiada. 
c. La evaluación dinámica, es considerada como el procedimiento más adecuado para el análisis del proceso de aprendizaje ya que los cambios específicos, en el funcionamiento cognitivo, tienen lugar durante el desarrollo de dicho proceso y debido a la influencia de la ayuda educativa que ha sido proporcionada. Desde la perspectiva sociocultural se propone que la finalidad de la evaluación no es la de obtener información cuantitativa, acerca de lo que el alumno/a discapacitado ha aprendido, sino la de conocer el potencial de aprendizaje de ese alumno/a en una actividad de colaboración conjunta. Según expone Kozulin (2000, p. 87), Vygotski (1986), sostenía que "la tarea de evaluación no sólo debe identificar los procesos cognitivos del niño, que están plenamente desarrollados, sino también los que se encuentran en proceso de desarrollo en el momento de la evaluación Este desarrollo depende de una interacción cooperadora entre el niño y el adulto que representa la cultura y que le ayuda a adquirir los instrumentos simbólicos necesarios para el aprendizaje”. En opinión de Kozulin (2000, págs. 88-89), la evaluación dinámica presenta las siguientes particularidades: los procesos cognitivos son muy modificables y, por esta razón, la tarea de evaluar consiste en determinar el grado de modificabilidad en lugar del nivel manifiesto de rendimiento, la evaluación interactiva, que incluye la fase de aprendizaje, proporciona una comprensión mejor de la capacidad de aprendizaje del niño que la ejecución sin ayuda y el objetivo de la evaluación es poner de manifiesto el potencial de aprendizaje del niño y proponer intervenciones psicopedagógicas orientadas a potenciar y hacer realidad ese potencial.

d. Intervención educativa de naturaleza mediatizada y compensatoria (Escoriza, 1998b): internalización de instrumentos socioculturales en actividades de colaboración conjunta. Según Kozulin (2000, p. 184) “Una misma función (por ejemplo, la memoria o la atención) aparece de dos formas totalmente diferentes si se percibe como una propiedad individual o si se percibe como el resultado de los procesos simbólicos de una cultura de los que un individuo se apropia y que, en cierto sentido, son supraindividuales. En consecuencia, el interés de la psicología educativa y escolar ya no recae tanto en identificar las aptitudes y las propensiones individuales de los estudiantes y se centra más en las actividades socialmente construidas que permiten desarrollar el potencial de aprendizaje y las funciones psicológicas superiores de los estudiantes”. La internalización de instrumentos psicológicos se configura, por tanto, como la característica principal de los procesos de enseñanzaaprendizaje. Al igual que con las personas, sin ningún tipo de discapacidad, el concepto de internalización de instrumentos psicológicos, en el contexto de actividades de colaboración 
conjunta con personas más expertas, es considerado como el principal mecanismo para promover el desarrollo en el caso de personas con alguna disfunción orgánica y/o psicológica.

e. La discapacidad es interpretada como un proceso durante el cual se van produciendo modificaciones o cambios cualitativos en el funcionamiento cognitivo. Los errores son considerados esenciales en el proceso de aprendizaje de la lectura en el sentido de que nos informan de cómo va evolucionando dicho proceso. Por esta razón, deben ser considerados como estados transitorios y no como estados permanentes causados por la incidencia de un déficit de mayor o menor gravedad. En cuanto a su evaluación, lo procedente es que sean identificados en el proceso de comprensión del discurso escrito.

Hasta aquí hemos procedido al análisis de uno de los elementos nucleares en los que se fundamenta la fuerte controversia, existente en la actualidad, acerca de la explicación de las dificultades específicas de aprendizaje del LE. Otro de los núcleos conflictivos, es identificable en la conceptualización del proceso de lectura: la lectura como una habilidad compleja, lenguaje integrado y constructivismo (Escoriza, 1996a, 1998a, 2003). Una de las cuestiones, objeto de polémicas continuadas, gira en torno a los posicionamientos discrepantes con respecto a la relevancia de los dos componentes de la lectura, la decodificación (identificación automatizada de palabras) y la comprensión (construcción del conocimiento), en el proceso de aprendizaje del LE. Asumir que la habilidad básica, en el proceso de lectura, es la identificación automatizada de las palabras implica otorgar una mayor relevancia al conocimiento de la forma del LE que al significado. El conocimiento fonémico se configura como el prerrequisito básico para que tenga lugar la decodificación y, las dificultades de aprendizaje del LE son atribuibles a un conocimiento fonémico insuficiente. Para Pressley (1997), por ejemplo, el fracaso en la lectura es debido a deficiencias en el conocimiento fonémico. Lyon (1995), al analizar la conceptualización de la dislexia, sostiene que la dislexia es un trastorno caracterizado por las dificultades en la decodificación de palabras aisladas y que, normalmente, es un reflejo de una insuficiencia en el procesamiento fonológico. Desde esta perspectiva, se considera que las dificultades deben ser evaluadas a nivel de identificación de palabras en base al supuesto de que la decodificación correcta de todas las palabras garantiza la comprensión del discurso escrito. Sin embargo, tanto el lenguaje integrado como el constructivismo son coincidentes en proponer que la comprensión del significado es más relevante que el conocimiento de la forma ya que la lectura es, esencialmente, un proceso de construcción del conocimiento y no una habilidad en la identificación de palabras. En consecuencia, las dificultades de aprendizaje del lenguaje escrito no pueden quedar reducidas 
a la decodificación automatizada de las palabras, sino que pueden ser observadas en cualquiera de los dos componentes de la actividad de lectura: la decodificación y la comprensión. La decodificación es considerada como un objetivo subsidiario de la comprensión, es decir, las dificultades que se generan a nivel de decodificación deben ser objeto de atención educativa solamente en aquellos casos en los que afecten de forma significativa a la comprensión. Las dificultades, en el componente comprensión, deberán ser evaluadas en el conocimiento estratégico que el alumno/a aplique para la construcción de representaciones mentales coherentes y estructuradas (Escoriza, 2003).

\begin{tabular}{|c|c|c|c|c|}
\hline Perspectiva DA & Modelo de DA & Hipótesis & $\begin{array}{l}\text { Conceptualización } \\
\text { de la lectura }\end{array}$ & Manifestación \\
\hline \multirow{11}{*}{ Neuropsicológica } & \multirow{4}{*}{$\begin{array}{l}\text { Médico: } \\
\text { Disfunción } \\
\text { Orgánica }\end{array}$} & Hemisferial & \multirow{11}{*}{$\begin{array}{l}\text { La Lectura como } \\
\text { una habilidad } \\
\text { Compleja: } \\
\text { * Componente de } \\
\text { Habilidades. }\end{array}$} & \multirow{11}{*}{$\begin{array}{l}\text { Dificultades en } \\
\text { Identificación de } \\
\text { Palabras. }\end{array}$} \\
\hline & & Cerebelar & & \\
\hline & & Magnocelular & & \\
\hline & & Genética & & \\
\hline & \multirow{5}{*}{$\begin{array}{l}\text { PCB: Déficit } \\
\text { cognitivo }\end{array}$} & Perceptivo & & \\
\hline & & Fonológico & & \\
\hline & & $\begin{array}{l}\text { Nombramiento } \\
\text { rápido }\end{array}$ & & \\
\hline & & Doble déficit & & \\
\hline & & Memoria & & \\
\hline & Conductual & $\begin{array}{l}\text { Práctica } \\
\text { insuficiente }\end{array}$ & & \\
\hline & Estratégico & $\begin{array}{l}\text { Déficit } \\
\text { Estratégico }\end{array}$ & & \\
\hline \multirow[b]{2}{*}{ Sociocultural } & \multirow[b]{2}{*}{ Constructivista } & $\begin{array}{l}\text { Experiencias con } \\
\text { el Lenguaje } \\
\text { Escrito }\end{array}$ & Lenguaje Integrado & $\begin{array}{l}\text { Dificultades } \\
\text { de Compren- } \\
\text { sión }\end{array}$ \\
\hline & & Mediacional & Constructivista & $\begin{array}{l}\text { - Dificultades } \\
\text { Decodificación. } \\
\text { - Dificultades } \\
\text { Comprensión. }\end{array}$ \\
\hline
\end{tabular}

Tabla 1. Esquema-guión para la explicación de las dificultades en el aprendizaje del lenguaje escrito y/o para la interpretación de las definiciones elaboradas acerca de la Dislexia. 
Análisis de las dificultades en la comprensión y aplicación del principio alfabético

\section{Conocimiento fonológico y dislexia.}

En el apartado anterior hemos descrito, de forma general, la situación tan problemática generada básicamente como consecuencia de la profunda controversia existente, a nivel explicativo, en dos ámbitos epistemológicos: conceptuación de las DA y conceptuación del proceso de lectura (ver tabla 1). El resultado es que, a pesar del volumen de investigaciones realizadas (la relación, entre conocimiento fonológico y lectura, viene siendo estudiada desde los años 70, Stahl y Murray, 1994; Escoriza, 1986), no existe aún una explicación unificada y concluyente acerca de las causas de las dificultades específicas en el aprendizaje del lenguaje escrito.

No obstante, podemos mencionar algunas propuestas sobre las que se está produciendo un cierto nivel de consenso entre los investigadores. Una de ellas es la que hace referencia a la relación entre déficit en el conocimiento fonológico y dislexia. En la investigación de la dislexia, existe una larga tradición defensora de la hipótesis perceptivo visual y que Pogorzelski y Wheldall (2002), la consideran como extensamente desacreditada ya que la investigación reciente ha identificado, como fundamental, un problema básico en el lenguaje o, de forma más específica, un problema de procesamiento fonológico: la presencia del déficit, en el procesamiento fonológico, conduce al fracaso para dominar el principio alfabético y, consecuentemente, a desarrollar una lectura automatizada y fluida. En la explicación de la dislexia, causada por un déficit fonológico, Frith (1999) afirma que hay una fuerte evidencia de que las dificultades, en la adquisición de la lectura, están relacionadas con dificultades en la aptitud para segmentar la secuencia sonora en unidades fonémicas y que, en las explicaciones de la dislexia, el déficit fonológico, es considerado como la causa proximal cognitiva común en todas ellas. Así, por ejemplo, en la definición de dislexia, propuesta por la Orton Dyslexia Society Research Comité (1994) (citada por Lyon, 1995, p. 9), se asume, entre otras cosas, que normalmente es el reflejo de insuficiencias en el procesamiento fonológico. En opinión de Frith (1999), la relevancia de la fonología, en el aprendizaje de la lectura, es asumida por la totalidad de los modelos actuales de la psicología cognitiva ya que el alfabeto está basado en la idea de que el lenguaje oral puede ser representado en unidades pequeñas y abstractas, los fonemas, los cuales pueden ser representados por los grafemas y, en consecuencia, la tarea principal que un niño tiene que dominar, cuando aprende a leer en un sistema alfabético, consiste en comprender cómo representar los sonidos orales mediante letras y cómo traducir con precisión el lenguaje oral al lenguaje escrito (y viceversa). Algunos 
de los supuestos básicos, en los que se fundamenta la relación entre conocimiento fonológico y aprendizaje del lenguaje escrito, son los siguientes (Escoriza, 2001 p. 148): el lenguaje escrito representa al lenguaje oral: leer supone traducir el lenguaje escrito al lenguaje oral, la comprensión del principio alfabético es necesaria para la identificación de palabras en un sistema alfabético, la lectura supone el acceso al léxico interno a partir de la representación gráfica del lenguaje oral y las dificultades evaluadas, en el proceso de identificación de palabras, pueden hacer menos operativo y funcional el proceso de decodificación y generar problemas en los procesos de comprensión del lenguaje escrito.

Para Howes et al., (2003), las dificultades de lectura son causadas por discapacidades específicas en las funciones del lenguaje implicadas en el procesamiento de los sonidos de las palabras. Según esta hipótesis, la dislexia surge de las deficiencias básicas en la capacidad para generar y mantener representaciones fonológicas y para manipular estas representaciones en la memoria de trabajo. Frith (1999), sostiene que, de todas las funciones en las que puede ser segmentado el lenguaje, el procesamiento de los sonidos del lenguaje oral ha sido indicada como el punto de conexión básico entre LO y LE, siendo las dificultades para segmentar la secuencia sonora las que están relacionadas con las dificultades para el aprendizaje de la lectura. Este tipo de dificultades son las que impiden lograr la competencia en el empleo de la estrategia alfabética y ello, a su vez, constituye un obstáculo para lograr el dominio de la estrategia ortográfica que es la que, según Frith (1999, 2002), permite el acceso automático al significado de las palabras escritas al igual que ocurre con las palabras orales. Catts et al., (2002), son contundentes al afirmar que actualmente, el candidato más relevante, para determinar las causas de la dislexia, son los problemas en el procesamiento fonológico: conocimiento fonológico y procesos fonológicos relacionados.

Los defensores de la hipótesis del déficit fonológico (Sterling et al., 1998; Cline, 2000; Cooke, 2002; Seymour y Duncan, 1997; Thomson, 1999; Frith, 1999, 2002; Tonnessen, 1997; Lyon, 1995; Howes et al., 2003; Shatschneider et al., 2002; Anthony y Lonigan, 2004; Stahl y Murray, 1994; Reynolds, Nicolson y Hambley, 2003; Vellutino y Scanlon, 2002; Zabell y Everatt, 2002, entre otros), fundamentan sus posicionamientos en la relación existente entre competencia metalingüística y aprendizaje de la lectura. De acuerdo con los resultados obtenidos por Steling et al., el déficit fonológico puede manifestarse de formas diversas (Sterling et al., (1998): confusiones en la memoria fonológica, elaborar representaciones fonológicas insuficientes o incorrectas en la memoria y deterioro de la base fonológica 
empleada para producir el output (sustitución de consonantes, omisión de fonemas, sustituciones lexicales y estructura fonémica no respetada). (Otros tipos de dificultades observadas, en los casos de dislexia, pueden verse, por ejemplo, en los trabajos de Cook, 2002; Paradice, 2001; la revisión efectuada por Pressley, 1997; Bryant y Bradley, 1985; Stahl y Murray, 1994; Torgesen et al., 1999; Pugh et al., 2000; Chard, Vaughn y Tyler, 2002).

\section{Dificultades en la comprensión y aplicación del principio alfabético}

El aprendizaje del lenguaje escrito, en un sistema alfabético, requiere tanto la comprensión del principio alfabético como su aplicación efectiva para la identificación de palabras. McCutchen et al., (2002), sostienen que existe un volumen importante de evidencia empírica favorable a la consideración de la comprensión del principio alfabético como un precursor crítico de la literidad. La comprensión del principio alfabético hace referencia al conocimiento explícito (Wright y Jacobs, 2003) de que los elementos gráficos representan a los correspondientes elementos del lenguaje oral o, en otros términos (Escoriza, 2001), a la comprensión de que las letras (identidad grafémica) representan a las unidades más abstractas del lenguaje oral y que las palabras tienen una estructura fonológica interna particular constituida por segmentos fonémicos individuales (identidad fonémica). De forma complementaria, a la comprensión del principio alfabético, Bryant y Bradley (1985) sugieren que los niños/as, con dificultades en la lectura, no necesariamente les falta un adecuado conocimiento fonológico sino que, más bien, tienen problemas en aplicar de forma flexible este conocimiento en la identificación de palabras. Igualmente, Greany, Tunmer y Chapman (1997), comprobaron que los niños/as, con dificultades en lectura demostraron un adecuado conocimiento de los ataques y rimas de las palabras y tenían buenas habilidades de segmentación pero que presentaban dificultades para aplicar este conocimiento en el proceso de identificación de palabras. El impacto más importante, de la debilidad en la aptitud para procesar la información fonológica, se manifiesta, según Torgesen et al., (1999), en las dificultades que experimentan los niños/as, en la comprensión y aplicación del principio alfabético a la hora de proceder a la decodificación de las palabras escritas. Para aprender a leer (Ehri, 2002), los niños/as deben adquirir el conocimiento del sistema alfabético y deben aprender a emplearlo para leer y escribir palabras. Vellutino y Scanlon (2002), consideran necesario que los niños, que aprenden a leer en una ortografía alfabética, deben comprender y adquirir el empleo funcional del principio alfabético. 
La comprensión del principio alfabético implica la internalización de una serie de contenidos diversos todos ellos referidos al conocimiento de las relaciones existentes entre LO y LE a nivel de estructura lexical, al ser considerado el procesamiento de los sonidos del lenguaje como la relación crítica existente entre ambos. Según Frith (1999), el principio alfabético está basado en la idea de que el LO puede ser representado por unidades pequeñas, los fonemas, que son representados mediante las letras y, por tanto, la tarea principal que un niño/a debe dominar, cuando está aprendiendo a leer, es la de comprender cómo los sonidos orales pueden y deben ser representados mediante las letras y cómo traducir el LE al LO. Para Silva y Alves (2003), la comprensión del principio alfabético supone: comprender la relación entre lenguaje oral y lenguaje escrito a nivel de segmentos fonémico-grafémicos y comprender la función notacional de la escritura y que esta función se hace operativa, en última instancia, en la relación LO-LE a nivel de los segmentos fonémico-grafémicos: que las letras constituyen un soporte concreto para la representación de los segmentos abstractos del LO (los fonemas), es decir, la escritura codifica los segmentos del LO.

McCutchen et al., (2002), mencionan como componentes del principio alfabético, a los dos siguientes: que el LO está compuesto de fonemas y que las letras representan a esos fonemas. Wright y Jacobs (2003) sostienen que el objetivo, de la intervención educativa, es el de focalizar la atención en los segmentos fonológicos de las palabras y en las reglas y principios de las correspondencias grafema-fonema tanto en la lectura como en la escritura de palabras promoviendo la comprensión del principio alfabético en sus tres componentes: comprender la estructura fonológica interna de la palabra oral, comprender que los cambios en la estructura interna de la palabra da lugar a cambios en su significado y comprender que, en la mayoría de los casos, los cambios en el significado lexical generan cambios en su estructura fonológica. Por nuestra parte (Escoriza, 2001), entendemos que la comprensión del principio alfabético supone:

A. En general, comprender que la palabra oral se puede segmentar en unidades cada vez más pequeñas (que es una totalidad segmentable; que tiene una estructura interna) y que existe una relación de cierta correspondencia entre estas unidades y los grafemas componentes de la palabra escrita que los representa. Cada palabra tiene su propia identidad fonémica, grafémica y semántica. La alteración de la forma (fonológica y/o grafémica) implica alteraciones en el significado. 
Análisis de las dificultades en la comprensión y aplicación del principio alfabético

B. En particular, supone comprender que, a la hora de establecer dicha correspondencia, son tres los componentes que deben ser tenidos en cuenta:

a. El número o cantidad de fonemas: para leer/escribir correctamente una palabra deben estar representados todos los fonemas de la palabra oral. Las palabras orales están compuestas de un número limitado y concreto de fonemas que requiere una representación grafémica igualmente limitada y concreta. El establecimiento de correspondencias fonémicografémicas correctas requiere, por tanto, que los fonemas sean representados en su totalidad mediante los grafemas necesarios y no añadir ni omitir ninguno de los segmentos fonémicos que definen la estructura sonora de la palabra oral.

b. El orden o secuenciación de los fonemas: para leer/escribir correctamente una palabra, los fonemas deben estar representados en el orden o secuencia que define la estructura de la palabra oral. Las palabras orales tienen una estructura interna propia y en la que los fonemas tienen un orden determinado y cuya secuencia debe ser respetada a la hora de concretar la secuencia grafémica adecuada para cada palabra oral.

c. La identidad de los fonemas: para leer/escribir correctamente una palabra, debe tenerse en cuenta la identidad fonémica y grafémica de cada uno de los segmentos sublexicales correspondientes. Las palabras orales están compuestas de unidades fonémicas que deben ser identificadas explícitamente en la secuencia sonora del lenguaje ya que definen la identidad fonológica de cada unidad lexical y que, en consecuencia, deben ser representados de forma conveniente por los grafemas correspondientes.

Las dificultades observadas, en la comprensión y aplicación del principio alfabético, las podemos categorizar en las siguientes estrategias fonológicas compensatorias que los alumnos/as suelen emplear para resolver el conflicto que se genera a la hora de establecer las necesarias correspondencias fonémico-grafémicas entre la estructura de la palabra oral y la palabra escrita (un análisis detallado de las categorías de dificultades, y de sus posibles explicaciones, puede verse en Escoriza y Boj, 1997):

a. Adición de fonemas. Mediante el empleo de esta estrategia, el alumno/a intenta resolver el problema que le plantea el establecimiento de correspondencias fonémicografémicas de palabras cuya estructura silábica presenta ataques formados por grupo consonántico y/o rimas compuestas por vocal y consonante. Las dificultades, en la comprensión y aplicación del primer componente del principio alfabético, se ponen de 
manifiesto al intercalar una vocal parásita interna de naturaleza fonética análoga al núcleo vocálico de la sílaba: la sílaba "fle” es representada como "fele".

b. Inversión en la secuenciación de los fonemas. Esta estrategia supone la alteración del orden de los fonemas a la hora de proceder a su representación grafémica. Constituye, por tanto, una manifestación de las dificultades en la comprensión y aplicación del segundo componente del principio alfabético: ordenación adecuada de la correspondencia fonémicografémica. Así, por ejemplo, la palabra “flor" es representada grafémicamente como "folr".

c. Omisión de fonemas. Es ésta una de las estrategias compensatorias más empleadas por los alumnos/as en los inicios del aprendizaje del lenguaje escrito. Su aplicación se manifiesta mediante la omisión de fonemas en las unidades intrasilábicas: supresión de un fonema del grupo consonántico en ataque silábico (por ejemplo, la palabra "libro" es escrita como "libo") y/o supresión del fonema consonántico en rima silábica (por ejemplo: la palabra "manzana” es escrita como "mazana").

d. Sustitución de fonemas. El fonema como unidad lingüística abstracta (no existe por sí mismo en la señal acústica) y los fenómenos de coarticulación o de transmisión en paralelo, han sido indicadas como las dos causas explicativas de las dificultades que se producen en la identificación de fonemas. Los fonemas (Share, 1995), son representaciones abstractas de familias de sonidos fonéticos que varían considerablemente en función del ritmo de pronunciación, entonación y, sobretodo, de la coarticulación o transmisión en paralelo. Una de las conclusiones, formuladas por Stuart (1986), es que hasta que no se haya adquirido el concepto de fonema, como unidad lingüística abstracta, el niño/a sólo podrá ser capaz de tratar y comprender las variantes superficiales, de la estructura sonora de la palabra, en términos de rasgos fonéticos. La manifestación, de este tipo de estrategia, suele presentar formas diversas. Una de ellas, consiste en la confusión fonética que se produce entre los fonemas “ $b$ ” " " $m$ ” en el contexto de la palabra (por ejemplo: la palabra "bombilla", aparece representada como "mommilla"; "bandera", como "dandera"). Para evitar los efectos no deseados, que generan este tipo de sustituciones fonémicas, en los intercambios comunicativos ordinarios, y cuando tenemos que deletrear una palabra cuya representación grafémica deseamos que sea correcta, solemos emplear el recurso de " $d$ " de Dinamarca, " $b$ " de Barcelona, " $m$ " de Murcia, o también aquello de "un billón, con b" para que quede claro que no estamos hablando de "millones" sino de una cifra muy superior (esta tarea, aparentemente sencilla, presenta un 
cierto grado de complejidad para las personas que tienen dificultades en la comprensión y aplicación del principio alfabético).

Las cuatro estrategias fonológicas compensatorias expuestas, todas ellas de naturaleza fonética, las consideramos como la manifestación de las dificultades que se pueden producir en la comprensión y aplicación de cada uno de los componentes del principio alfabético (tabla 2): las adiciones $y$ omisiones de fonemas, son indicativas de que el alumno/a no ha comprendido el primer componente (número de fonemas necesarios para leer/escribir una palabra), las inversiones de fonemas evidencian las dificultades relacionadas con el segundo componente (orden, secuencia o estructura fonémica de la palabra) y las sustituciones, se generan debido a los problemas que plantea la aplicación del tercer componente (la identidad de los fonemas).

\begin{tabular}{|c|c|c|c|c|c|c|}
\hline $\begin{array}{l}\text { Componentes del } \\
\text { Principio } \\
\text { Alfabético }\end{array}$ & $\begin{array}{l}\text { Estrategia } \\
\text { Fonológica } \\
\text { Compensatoria }\end{array}$ & $C-V$ & $\begin{array}{l}\text { uctura silábica } \\
C-V+C \quad C C-V\end{array}$ & Ataque & $\begin{array}{l}\text { rasilábica } \\
\text { Rima }\end{array}$ & $\begin{array}{l}\text { Fonemas } \\
\text { Específicos }\end{array}$ \\
\hline \multirow[t]{2}{*}{ a. Número } & Adición & & & & & \\
\hline & Omisión & & & & & \\
\hline b. Secuencia & Inversión & & & & & \\
\hline c. Identidad & Sustitución & & & & & \\
\hline Totales & & & & & & \\
\hline
\end{tabular}

Tabla 2. Procedimiento para la evaluación del conocimiento fonológico.

En su conjunto, tanto los componentes del principio alfabético como las estrategias fonológicas enunciadas, nos permiten interpretar la dislexia o las dificultades específicas en el aprendizaje del lenguaje escrito, en los términos siguientes:

a. Se caracteriza por las dificultades para la identificación de palabras de forma fluida/automatizada y correcta/precisa (identidad fonémica, grafémica y semántica) y que afectan y están referidas al componente decodificador del LE: grado de desarrollo de la competencia literada a nivel lexical o de palabras.

b. Estas dificultades tienen su origen en una comprensión insuficiente de los diversos componentes del principio alfabético y/o en una aplicación incorrecta de dichos componentes, debido a experiencias de literidad limitadas y/o inadecuadas o, bien, como expone Kozulin (2000), refiriéndose a las experiencias de aprendizaje mediatizado, una cantidad insuficiente 
de mediación y unas condiciones que hacen que una cantidad o un tipo de mediación normales sean insuficientes o inadecuadas. Para Bryant y Bradley (1985), las dificultades de lectura, no deben ser interpretadas como déficits en una habilidad, sino como una habilidad que aún no se ha desarrollado y que, por tanto, no es cuestión de déficit sino de desarrollo (o de nivel en la competencia literada, Escoriza, 1996c, 1998a). Los problemas generados, en la comprensión y/o aplicación del principio alfabético, pueden ser observados en el empleo de las estrategias fonológicas compensatorias enunciadas (adición, inversión, omisión y supresión de fonemas), indicativas todas ellas del progreso realizado por el alumno/a en el proceso de internalización de la conciencia fonológica.

c. La consecuencia, es una competencia de bajo nivel en el empleo del lenguaje escrito, como instrumento psicológico multifuncional, en su medio sociocultural (instrumento de aprendizaje, comunicativo y regulador).

Para la evaluación, del proceso de internalización de la conciencia fonológica, se han venido aplicando dos procedimientos generales: la evaluación psicométrica y la evaluación dinámica. En nuestro caso proponemos el segundo sistema, al que Scheneider y Ganschow (2000) consideran como un método adecuado para evaluar el potencial intelectual y que está fundamentado en el supuesto básico de que las personas pueden modificar y mejorar sus procesos de aprendizaje si participan en actividades interactivas con profesores/as que le proporcionan experiencias adecuadas de aprendizaje mediatizado. En la evaluación dinámica (Scheneider y Ganschow, 2000), adquiere una especial relevancia: el papel del profesor/a como mediador y facilitador del proceso de aprendizaje del alumno/a, la integración/interrelación de evaluación y enseñanza y la focalización en el proceso más que en el producto.

Ya hemos indicado en otro momento (Escoriza, 1998b) que la evaluación, como proceso, supone dar respuesta a cuatro cuestiones: Qué, cómo, cuándo y porqué evaluar. Responder a estas cuestiones, implica, entre otras cosas, concretar cuál es el verdadero sujeto de aprendizaje si el alumno/a en solitario o, tal y como propone Kozulin (2000), la totalidad integradora que incluye al niño/a, al adulto experto y al instrumento simbólico proporcionado por una sociedad dada. En consecuencia, las dificultades de aprendizaje del LE deben ser analizadas y evaluadas en el contexto dinámico definido por la unidad formada por aprendiz/experiencias de literidad/profesor/a. 
En el caso de las dificultades de aprendizaje, Dwairy (2004), formula las siguientes críticas a la evaluación mediante el empleo de los tests: Aprender a leer y a escribir es un proceso integrativo en el que las funciones cognitivas operan de forma simultánea y diferente a como operan en los tests cognitivos. La evaluación separada, de funciones psicológicas específicas, no se corresponde con los procesos integrativos cognitivos que tienen lugar en el aprendizaje. Normalmente, los tests evalúan lo que ha sido aprendido y no lo que puede ser aprendido cuando otra persona experta le proporciona enseñanza y guía competente y eficaz. Lo que debe ser evaluado es el progreso (la ejecución potencial) durante el proceso y no el producto en términos de rendimiento (ejecución actual). En esta misma línea discrepante, podemos situar los comentarios que formulan Wearmouth y Reid (2000), al afirmar que los tests no pueden indicar estrategias adecuadas de intervención ya que las puntuaciones no proporcionan detalles de lo que el niño sabe o no sabe, ni evidencian los procesos que están implicados en las dificultades del niño (un análisis crítico es el efectuado también por Weaver, 1990).

Teniendo en cuenta las consideraciones que acabamos de exponer, el sistema de evaluación que proponemos presenta las siguientes características (tablas 2,3 y 4):

a. Qué evaluar: el progreso del alumno/a cuando participa en actividades de literidad funcionales y propositivas, guiadas por la ayuda educativa que le proporciona el profesor/a. En concreto, y relacionado con la problemática que estamos analizando, procede evaluar el desarrollo de la competencia del alumno con respecto a la manipulación explícita de representaciones fonológicas de los segmentos fonémicos que forman parte de la estructura sonora de la palabra oral: comprensión y aplicación funcional de cada uno de los componentes del principio alfabético.

b. Cómo evaluar: el procedimiento de evaluación que sugerimos (Escoriza, 2001, 2002), tiene, como unidad de análisis, el contexto dinámico definido por alumno/ainstrumento psicológico-profesor/a, y supone un proceso tendente a obtener información con respecto al progreso del alumno/a (ejecución potencial) en la comprensión y aplicación funcional de los componentes del principio alfabético.

c. Porqué evaluar: los datos obtenidos, mediante el proceso de evaluación, deben tener como finalidad concretar con precisión las necesidades educativas más relevantes del alumno/a. La necesaria e ineludible integración, entre evaluación y enseñanza, nos conduce al 
empleo de esta información para diseñar y desarrollar un proceso de intervención educativa contingente con las necesidades educativas identificadas. En nuestro caso (ver tabla 2), dicha información nos debe permitir determinar qué componente o componentes del principio alfabético requieren un tratamiento educativo diferente y qué tipo de contenidos ( estructura silábica, unidad intrasilábica, unidades fonémicas específicas) son los que debemos seleccionar como los más pertinentes y cómo procede secuenciarlos, en función de los objetivos educativos previstos. En el diseño, del proceso de intervención educativa, el objetivo general es el de promover la comprensión y aplicación del principio alfabético y los objetivos específicos estarán referidos a promover la comprensión y aplicación del componente concreto en el que observemos que el alumno/a sigue empleando cualquiera de las estrategias fonológicas compensatorias indicadas. En cuanto a los contenidos, debemos proponer actividades de literidad (identificación, supresión, adición, segmentación, integración, secuenciación de fonemas, etc.) en las que el alumno/a tenga la oportunidad de realizar operaciones cognitivas que supongan la manipulación de fonemas en aquellas estructuras silábicas y unidades intrasilábicas en las que el alumno/a manifiesta una comprensión insuficiente o una aplicación inadecuada.

\begin{tabular}{|c|c|c|c|c|c|c|c|c|c|}
\hline \multicolumn{2}{|c|}{$\begin{array}{l}\text { Componentes del } \\
\text { Principio } \\
\text { Alfabético } \\
\end{array}$} & \multicolumn{2}{|c|}{$\begin{array}{l}\text { Estrategia } \\
\text { Fonológica } \\
\text { Compensatoria }\end{array}$} & $C-V$ & ictura sila & $C C-V$ & Unidac & rasilábica & \multirow[t]{2}{*}{$\begin{array}{l}\text { Fonemas } \\
\text { Específicos }\end{array}$} \\
\hline \multirow[t]{2}{*}{ a. Número } & \multirow[t]{2}{*}{1} & Adición & & & & & & & \\
\hline & & Omisión & 1 & & 1 & & & 1 & $\mathrm{~m}$ \\
\hline b. Secuencia & & Inversión & & & & & & & \\
\hline c. Identidad & 2 & Sustitución & 2 & 2 & & & 2 & & $\mathrm{~B}$ \\
\hline Totales & & & & & & & & & \\
\hline
\end{tabular}

Tabla 3. Ejemplo 1 del Procedimiento que se puede seguir para la evaluación del conocimiento fonológico de un alumno/a concreto: Palabra escrita, "momilla", en lugar de bombilla 


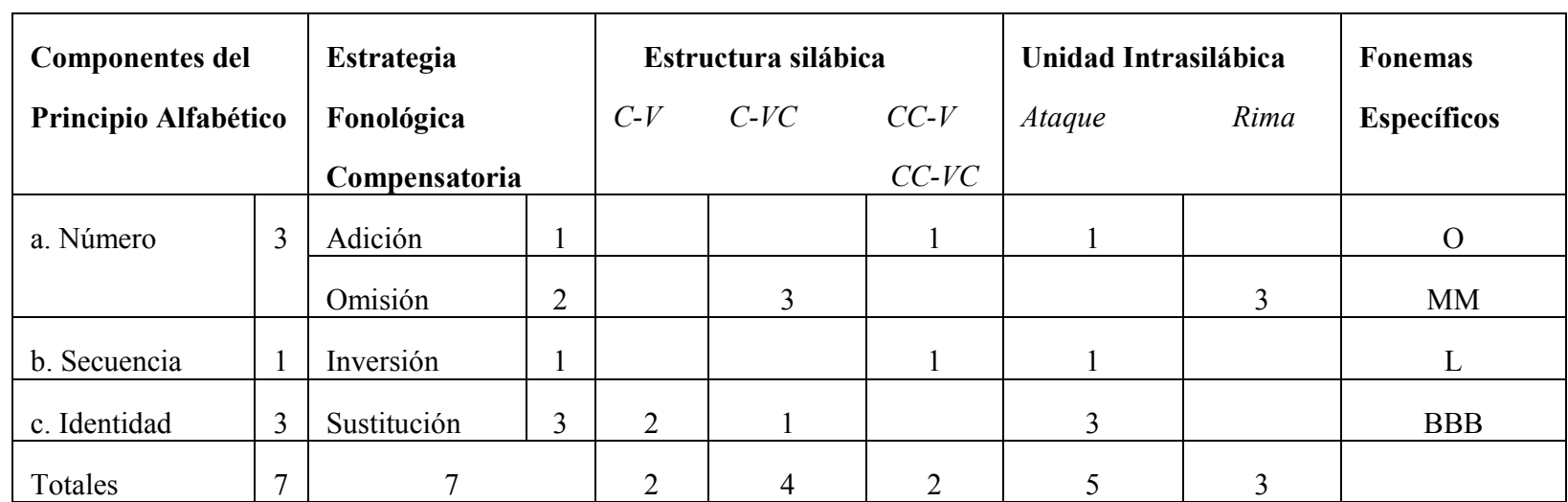

Tabla 4. Ejemplo del Procedimiento completo para la evaluación del conocimiento fonológico. Palabras escritas: "momilla" en lugar de bombilla, "folor" en lugar de flor, "madera" en lugar de bandera, "felcha" en lugar de flecha.

Finalmente, unas breves consideraciones con respecto a la naturaleza y orientación de los procesos de intervención educativa (Escoriza y Boj, 1992abcd). Las recomendaciones que han sido formuladas y los programas diseñados presentan diferencias profundas. Por una parte, se defiende la enseñanza descontextualizada del conocimiento fonológico en base a la relevancia que se le otorga a la recodificación fonológica y a la automatización de la decodificación en la identificación de palabras (los programas elaborados guardan una estrecha relación con las hipótesis del déficit fonológico y del doble déficit, Sterling et al., 1998; Cooke, 2002; Howes et al., 2003; Frith, 1999; Catts et al., 2002; Sodoro et al., 2002; Chard, Vaughn y Tyler, 2002; entre otros). Algunos ejemplos, de este tipo de propuestas, son los estudios realizados por Reason y Morfidi (2001), Vellutino y Scanlon (2002), Slocum, O’Connor y Jenking (1993), Torgesen et al., (1999), Brooks y Weeks (1998), Seymour y Duncan (1997) etc. y que, en grado variable, están basadas en las premisas en las se fundamenta la conceptualización de la lectura desde la perspectiva lineal (Escoriza, 1996a; Weaver, 1990; Pressley, 1999). Por otra, el énfasis recae en la enseñanza contextualizada del LE (lenguaje integrado, constructivismo, Escoriza 1996a, 2003), dándole prioridad a las experiencias funcionales y propositivas de literidad (conocimiento de las funciones del lenguaje escrito) y no al conocimiento de la forma (Escoriza y Boj, 1991; Weaver, 1990; Bergeron, 1990; Pressley, 1999). Vygotski (en Kozulin, 2000, p. 34) recomendaba iniciar la enseñanza del LE, pidiendo a los niños que designen ciertos objetos mediante pictogramas y signos y, una vez adquirida esta función simbólica esencial, se debería pasar del simbolismo 
de primer orden, que emplea signos para representar el contenido de una oración, a un simbolismo de segundo orden, que emplea letras para representar las palabras, es decir, enseñar la función de la simbolización antes de instruir en las técnicas específicas del LE. Pressley (1999), comentando el lenguaje integrado, afirma que esta interpretación del LE otorga una mayor primacía al desarrollo natural de la alfabetización que al desarrollo basado en la enseñanza de las habilidades básicas de lectura y que, en consecuencia, en las clases donde se aplica, la enseñanza de dichas habilidades solamente se lleva a cabo cuando determinados alumnos/as lo necesitan y únicamente en el contexto de la lectura y escritura y no como un punto esencial de la enseñanza. Las experiencias literadas tienen prioridad con respecto a la enseñanza directa de la decodificación. Mientras que el primer tipo de propuestas se centran, en general, en la palabra o en el fonema como unidad lingüística, el segundo propone centrar la intervención en el proceso y en el significado, en las actividades de lectura y escritura como procesos simultáneos e interactivos. Edwards (2003), indica que varios estudios han demostrado que la escritura puede facilitar la lectura, el análisis de las palabras, etc. ya que la práctica, en la escritura de palabras, puede ser una forma concreta de reforzar el conocimiento fonémico y facilitar la lectura de palabras. En este sentido, Edwards menciona los trabajos de Treiman, relativos a la eficacia de la escritura para promover el análisis de las palabras orales y el conocimiento de cómo estos sonidos y las palabras son representados mediante el LE. Según indica Edwards (2003), los resultados obtenidos sugieren que los niños/as, con dificultades de aprendizaje, necesitan participar en actividades en las que el principio alfabético pueda ser internalizado de forma más comprensiva y funcional de lo que lo pueda ser mediante los métodos de tipo fonético. Enseñar, mediante las prácticas de escritura, la aplicación funcional del principio alfabético, es considerado como una forma de aprendizaje más significativa y motivadora, que el aprendizaje de fonemas aislados, ya que la escritura se caracteriza por ser una actividad global integrativa que, necesariamente, debe ser realizada en una situación discursiva. En la situación discursiva se dan todas las condiciones que van a posibilitar la transición de la escritura alfabética a la escritura ortográfica que es la que, en definitiva, caracteriza a la persona que no tiene ningún tipo de dificultades a nivel del componente decodificador. Para Lundberg (2002), el uso productivo de una escritura alfabética, requiere un conocimiento explícito de los fonemas y un control consciente de estas unidades, lo que debe permitir el que sean manipulados, subtituidos y recombinados (esta propuesta guarda una estrecha relación con la teoría evolutiva elaborada por U. Frith, en la que recomienda promover la estrategia alfabética mediante la escritura, con la finalidad de 
que se produzca la transición de la lectura logográfica a la lectura ortográfica). Promover la comprensión y aplicación funcional del principio alfabético, promover la transformación del conocimiento alfabético en conocimiento ortográfico, hacer operativa la decodificación, son todos ellos objetivos que pueden ser logrados mediante experiencias de literidad funcionales y propositivas (actividades de escritura y de lectura en situación discursiva).

A modo de criterios generales, a tener en cuenta, a la hora de diseñar un proceso de intervención educativa, orientado a promover la comprensión y aplicación funcional del principio alfabético, sugerimos los siguientes: evitar la enseñanza de habilidades aisladas o descontextualizadas (o como propone Bergeron, 1990: evitar las secuencias de habilidades para organizar la instrucción), considerar al LO y al LE, a la lectura y a la escritura como actividades complementarias, simultáneas, interactivas, transaccionales, otorgar una especial relevancia a las prácticas de escritura, promover el concepto de palabra como unidad lingüística que tiene una identidad propia (fonémica, grafémica, semántica) y la internalización de estrategias alternativas y complementarias para identificar palabras no familiares encontradas en los discursos escritos (Vellutino y Scanlon, 2002; También podríamos hacer mención al modelo interactivo compensatorio de Stanovich), seleccionar y secuenciar los contenidos y actividades en función de los objetivos educativos previstos. En nuestro caso, tal y como hemos indicado anteriormente, el objetivo educativo es muy claro y concreto (tabla 2): promover la comprensión y aplicación funcional de todos los componentes del principio alfabético o de alguno/s de los tres componentes En cuanto al tipo de actividades, seleccionar y secuenciar todas aquellas en las que: se produzca una relación directa entre forma y significado (comprender que los cambios en la forma generan cambios en el significado), supongan la comprensión de que las palabras están compuestas de segmentos fonémicos internos y la manipulación de representaciones fonológicas (segmentación, adición, supresión, identificación, síntesis, etc. de dichos segmentos (para Seymour y Duncan, 1997, la cuestión básica consiste en la división de las palabras en fonemas (unidades lingüísticas abstractas díficiles de percibir y de identificar), y que requieran el establecimiento de correspondencias entre fonemas y grafemas (que las palabras están compuestas de letras, que las letras son representaciones de unidades sonoras, que todas la letras y sus posiciones son importantes. Seymour y Duncan, 1997), consideran que, el problema clave a resolver, es la forma en la que las letras representan a los fonemas), etc. 
Para realizar las actividades, indicadas anteriormente, emplear las tareas que han sido estudiadas y aplicadas con la finalidad de promover el conocimiento fonémico (Escoriza, 1990-1991, Escoriza y Boj, 1992a, 1997): tarea de Elkonin, recomendada especialmente en el caso del primer componente del principio alfabético, las tareas de Slingerland y Stuart, para el segundo y tercer componente, combinación de dos o tres tareas (Elkonin, Slingerland y Stuart) en el caso de que las necesidades educativas del alumno/a así lo aconsejen y seleccionar (ver Escoriza y Boj, 1997 y Defior, 1996) las tareas, que se consideren más pertinentes para promover, de forma específica, la comprensión de cada uno de los tres componentes del principio alfabético o que se pueden relacionar de forma directa con cada una de las cuatro estrategias compensatorias: adición, omisión, inversión y sustitución de fonemas. Uno de los supuestos, puede ser del tipo siguiente: "Hemos observado, en las producciones escritas de un alumno/a, palabras escritas en las se han añadido fonemas innecesarios (folor, en lugar de flor) o ha sido omitido alguno de los fonemas necesarios, a la hora de proceder a su representación grafémica (badera, en lugar de bandera). En este caso, consideramos que tiene dificultades en la comprensión y aplicación del primer componente del principio alfabético (número o cantidad de fonemas). La primera tarea pertinente, a seleccionar es la de Elkonin (después podemos combinarla, por ejemplo, con la de Slingerland) y seguidamente debemos seleccionar, de las tareas expuestas en Escoriza y Boj, (1997) o Defior, (1996), las que consideremos más pertinentes teniendo en cuenta el objetivo educativo previsto y su manifestación mediante el empleo de las dos estrategias fonológicas aplicadas: adición y omisión".

\section{Referencias}

Anthony, J.L. y Lonigan, Ch.J. (2004). The nature of phonological awareness: converging evidence from four studies of preschool and early grade school children. Journal of Educational Psychology, 96(1), 43-55.

Bergeron, B.S. (1990). What does the term whole language mean?. Constructing a definition from the literature. Journal of Reading Behavior, XXII, 4.

Brooks, P.L. y Weeks, J.A. (1998). A comparison of the responses of dyslexic, slow learning and control children to different strategies for teaching spellings. Dyslexia, 4, 212-222. 
Análisis de las dificultades en la comprensión y aplicación del principio alfabético

Bryant, P.E. y Bradley, L. (1985). Children's reading problems. Oxford: Basil Blackwell.

Burden, B. (2002). A cognitive approach to dyslexia: learning styles and thinking skills. En G. Reid y J. Wearmouth (eds.), Dyslexia and Literacy. Theory and Practice, 271-283. Chichester: John Wiley and Sons.

Catts, H.W. et al., (2002). The role of speed of processing, rapid naming, and phonological awareness in reading achievement. Journal of Learning Disabilities, 35(6), 510-525.

Chard, D.J., Vaughn, Sh. y Tyler, B-J. (2002). A synthesis of research on effective interventions for building reading fluency with elementary students with learning disabilities. Journal of Learning Disabilities, 35(5), 386-406.

Cline, T. (2000). Multilingualism and dyslexia: challenges for research and practice. Dyslexia, $6,3-12$.

Cole, M. y Griffin, P (1989). Una aproximación socio-histórica a la re-mediación. Comunicación, Lenguaje y Educación, 3-4, 89-100.

Cooke, A. (2002). Case study: a virtual non-reader achieves a degree. Dyslexia, 6, 102-115.

Coon, K.B., Waguespack, M.M. y Polk, M.J. (1994). Dyslexia screening instrument. S. Antonio, TX: Psychological Corp.

Crombi, M. (2002). Dealing with diversity in the primary classroom-a challenge for the class teacher. En G. Reid y J. Wearmouth (eds.), Dyslexia and Literacy. Theory and Practice, 229-240. Chichester: John Wiley and Sons.

Daniels, H. (2003). Vygotsky y la Pedagogia. Barcelona: Paidós.

Dean, E.C., Howell, J. y Alder, B. (1998). The competencies underlying metaphonological processing in pre-literate children. Dyslexia, 4, 181-196.

Defior, S. (1996). Una clasificación de las tareas utilizadas en la evaluación de las habilidades fonológicas y algunas ideas para su mejora. Infancia y Aprendizaje, 73, 49-63.

Durkin, C. (2000). Dyslexia and bilingual children - does recent research assist identification?. Dyslexia, 6, 248-267. 
Dwairy, M. (2004). Dynamic approach to learning disability assessment: DLD test. Dyslexia, $10,1-23$.

Eckert, M.A. y Leonard, Ch.M. (2000). Structural imaging in dyslexia: the planum temporale. Mental Retardation and Developmental Disabilities Research Reviews, 6, 198-206.

Edwards, L. (2003). Writing instruction in kindergarten: examining an emerging area of research with writing and reading difficulties. Journal of Learning Disabilities, 36(2), 136-148.

Ehri, L.C. (2002). Reading processes, acquisition, and instructional implications. En G. Reid y J. Wearmouth (eds.), Dyslexia and Literacy. Theory and Practice, 167-185 Chichester: John Wiley and Sons.

Ellis, A.W., McDougall, S.J.P. y Monk, A.F. (1997). Are dyslexics different? III. Of course they are. Dislexia, 3, 2-8.

Escoriza, J. (1986). Madurez lectora: predicción, evaluación e implicaciones educativas. Barcelona: Promociones Publicaciones Universitarias.

Escoriza, J. (1988). La intervención psicopedagógica en el campo de la lectura. Revista Galega de Psicopedagogía, 1, 121-131.

Escoriza, J. (1990). Origen y supuestos básicos del enfoque psicolinguístico de la lectura. Revista de Logopedia, Foniatría y Audiología, X(4), 192-200.

Escoriza, J. (1990-1991). Conocimiento explícito de la estructura fonológica del lenguaje y adquisición de la lectura. Revista Galega de Psicopedagogía, 4-5(4), 55-65.

Escoriza, J. (1991). Niveles del conocimiento fonológico. Revista de Psicología General y Aplicada, 44, 269-276.

Escoriza, J. (1996a). El proceso de lectura: aspectos teórico-explicativos. En J.Escoriza, A. Barca, J. González y R. González (Eds.), Psicología de la Instrucción, 59-88. Barcelona: Ediciones Universitarias de Barcelona. 
Análisis de las dificultades en la comprensión y aplicación del principio alfabético

Escoriza, J. (1996b). Psicopedagogía del lenguaje escrito: la lectura. En J.Escoriza, A. Barca, J. González y R. González (Eds.), Psicología de la Instrucción, 89-132. Barcelona: Ediciones Universitarias de Barcelona.

Escoriza, J. (1996c). Educación de la literidad. Revista de Logopedia, Foniatría y Audiología, XVII(1), 39-46.

Escoriza, J. (1998a). ¿Dificultades en el proceso de aprendizaje de la lectura o niveles en el desarrollo de la competencia literada?. Revista Galega de Psicopedagogía, 10(14-15), 131-153.

Escoriza, J. (1998b). Conocimiento psicológico y conceptualización de las dificultades de aprendizaje. Barcelona: Edicions Universitat de Barcelona.

Escoriza, J. (2001). Evaluación educativa de las dificultades de lectura y escritura a nivel de conciencia fonológica. En A. Miranda (Comp.), Déficit de atención y Dificultades de Aprendizaje, 147-160. Málaga: Aljibe.

Escoriza, J. (2002). Análisis del campo de las Dificultades de Aprendizaje desde una perspectiva internacional. En J. Escoriza (Coord.), Dificultades de Aprendizaje: Contenidos teóricos y actividades prácticas, 83-101. Barcelona: Edicions Universitat de Barcelona.

Escoriza, J. (2003). Evaluación del conocimiento de las Estrategias de Comprensión Lectora. Barcelona: Edicions Universitat de Barcelona.

Escoriza, J. y Gras, M. (1986). Errores en la escritura a nivel de palabra. En J. Escoriza y M. Gras (eds.), Bases psicológicas de los procesos de lectura y escritura, 105-135. Barcelona: PPU.

Escoriza, J. y Boj, C. (1991). Secuenciación del conocimiento metalingüístico en el proceso de enseñanza del lenguaje escrito. Revista de Psicología de la Educación, 3(8), 31-48.

Escoriza, J. y Boj, C. (1992a). Transición del nivel fonético al nivel fonético en los inicios del aprendizaje del lenguaje escrito. Revista Galega de Psicopedagogía, 6-7, 177-205.

Escoriza, J. y Boj, C. (1992b). Intervención educativa a nivel de comprensión del principio alfabético (I). Revista de Logopedia, Foniatría y Audiología ,XII(1), 25-31. 
Escoriza, J. y Boj, C. (1992c). Intervención educativa a nivel de comprensión del principio alfabético (II). Revista de Logopedia, Foniatría y Audiología ,XII(2), 104-110.

Escoriza, J. y Boj, C. (1992d). Procesos y estrategias en los inicios del aprendizaje del lenguaje escrito. Implicaciones instruccionales. Revista Galega de Psicopedagoxía, 67, 83-94.

Escoriza, J. y Boj, C. (1996). Explicación del proceso de lectura y criterios de intervención educativa. Revista Galega de Psicopedagogía, 13(9), 43-56.

Escoriza, J. y Boj, C. (1997). Psicopedagogía de la escritura. Barcelona: Llibreria Universitaria de Barcelona.

Escoriza, J. y Boj, C. (1998).Incidencia de las explicaciones diferenciales del proceso de lectura en la interpretación de las dificultades en el proceso de aprendizaje del lenguaje escrito. Revista de Psicodidáctica, 6, 15-32.

Fawcett, A. (2002). Dyslexia and literacy: key iusses for research. En G. Reid y J. Wearmouth (eds.), Dyslexia and Literacy. Theory and Practice, 11-28. Chichester: John Wiley and Sons.

Frith, U. (1999). Paradoxes in the definition of dyslexia. Dyslexia, 5, 192-214.

Frith, U. (2002). Resolving the paradoxes of dyslexia. En G. Reid y J. Wearmouth (eds.), Dyslexia and Literacy. Theory and Practice, 69-83. Chichester: John Wiley and Sons.

Gang, M. Siegel, L.S. (2002). Sound-symbol learning in children with dyslexia. Journal of Learning Disabilities, 35(2), 137-151.

Gindis, B. (1995). The social/cultural implication of disability: Vygotsky's paradigm for special education. Educational Psychologist, 30(2), 77-81.

Goswami, U. (2000). Phonological representations, reading developmental and dyslexia: towards a cross-linguistic theoretical framework. Dyslexia, 6, 133-151.

Greaney, K.T., Tunmer, W. y Chapman, J.W. (1997). The use of rime-based orthographic analogy training as an intervention strategy for reading-disabled children. En B. 
Análisis de las dificultades en la comprensión y aplicación del principio alfabético

Blachman (ed.), Foundations of reading acquisition and dyslexia: Implications for early intervention (327-345). Mahwah, NJ: Lawrence Erlbaum Associates.

Hagtvet, B.E. (1997). Phonological and linguistic-cognitive precursors of reading abilities. Dyslexia, 3, 163-177.

Hatcher, J. y Snowling, M.J. (2002). The phonological representations hypothesis of dyslexia: from theory to practice. En G. Reid y J. Wearmouth (eds.), Dyslexia and Literacy. Theory and Practice, 45-68. Chichester: John Wiley and Sons.

Henry, M.K. (1997). The decoding/spelling curriculum: integrated decoding and spelling instruction from pre-school to early secondary school. Dyslexia, 3, 178-189.

Howes, N-L. et al., (2003). Children with dyslexia. Journal of Learning Disabilities, 36(3), 229-245.

Kelly, S.W., Griffiths, S. y Frith, U. (2002). Evidence for implicit sequence learning in dyslexia. Dyslexia, 8, 43-52.

Knight, D.F. y Hynd, G.W. (2002) The neurobiology of dyslexia. En G. Reid y J. Wearmouth (eds.), Dislexia and Literacy. Theory and Practice, 29-43. Chichester: John Wiley and Sons.

Kozulin, A. (2000). Instrumentos psicológicos. La educación desde una perspectiva sociocultural. Barcelona: Paidós.

Lovett, M.W. et al., (1994). Treating the core deficits of developmental dyslexia: evidence of transfer of learning after phonologically- and strategy-based reading training programmes. Developmental Psychology, 30, 805-822.

Lundberg, I. (2002). The child's route into reading and what can go wrong. Dyslexia, 8, 1-13.

Lyon, G.R. (1995). Toward a definition of dyslexia. Annals of Dyslexia, 45, 3-27.

Lyster, S-A.H. (1998). Preventing reading failure: a follow-up study. Dyslexia, 4, 132-144.

Martin, F., Pratt, Ch. y Fraser, J. (2000). The use of orthographic and phonological strategies for the decoding of words in children with developmental dyslexia and average readers. Dyslexia, 6, 231-247. 
McCutchen, D. et al., (2002). Beginning literacy: links among teacher knowledge, teacher practice, and student learning. Journal of Learning Disabilities, 35(1), 69-86.

McNulty, M.A. (2003). Dyslexia and the life course. Journal of Learning Disablities, 36(4), 363-381

Miles, E. (1997). Implications for teachers of dyslexics of some recent phonological research. Dyslexia, 3, 157-162

Newman, J.M. (1985). Insights from recent reading and writing research and their implications for the developing whole language curriculum. En JM. Newman (Ed.), Whole language. Theory in use, 1-36. Portsmouth: Heineman.

Nicolson, R.I. y Fawcett, A.J. (1999). Developmental dyslexia: the role of the cerebellum. Dyslexia, 5, 155-177.

O’Connor, R.E., Slocum, T.A. y Jenkins, J.R. (1995). Transfer among phonological tasks in kindergarten: essential instructional content. Journal of Educational Psychology, $87(2), 202-217$.

Olson, R.K. (2002). Dyslexia: nature and nurture. Dyslexia, 8, 143-159.

Paradice, R. (2001). An investigation into the social construction of dyslexia. Educational Psychology in Practice, 17(3), 213-225.

Perin, D. (1997). Workplace literacy assessment. Dyslexia, 3, 190-200.

Pogorzelski, S. y Wheldall, K. (2002). Do differences in phonological processing performance predict gains made by older low-progress readers following intensive literacy intervention?. Educational Psychology, 22(4), 413-427.

Pressley, M. (1999). Cómo enseñar a leer. Barcelona: Paidós.

Proctor, B. y Prevatt, F. (2003). Agreement among four models used for diagnosing learning disabilities. Journal of Learning Disabilities, 36(5), 459-466.

Pugh, K.R. et al., (2000). Functional neuroimaging studies of reading and reading disability. Mental Retardation and Developmental Disabilities Research Reviews, 6, 207-213. 
Análisis de las dificultades en la comprensión y aplicación del principio alfabético

Reason, R. (2002). From assessment to intervention: the educational psychology perspective. En G. Reid y J. Wearmouth (eds.), Dyslexia and Literacy. Theory and Practice, 187200. Chichester: John Wiley and Sons.

Reason, R. y Morfidi, E. (2001). Literacy difficulties and single-case experimental design. Educational Psychology in Practice, 17(3), 227-244.

Reynolds, D., Nicolson, R.I. y Hambly, H. (2003). Evaluation o fan exercise-based treatment for children with reading dificulties. Dyslexia, 9. 48-71.

Robertson, J. y Bakker, D.J. (2002). The balance model of reading and dyslexia. En G. Reid y J. Wearmouth (eds.), Dyslexia and Literacy. Theory and Practice, 99-114. Chichester: John Wiley and Sons.

Schatschneider, Ch. et al., (2002). Relationship of rapid automatized naming and phonological awareness in early reading development: Implications for the doubledeficit hypothesis. Journal of Learning Disabilities, 35(3), 245-256.

Seymour, Ph.H.K. y Duncan, L.G. (1997). Small versus large unit theories of reading acquisition. Dyslexia, 3, 125-134.

Shapiro, B.K. (2001). Specific reading disability: a multiplanar view. Mental Retardation and Developmental Disabilities Research Reviews, 7, 13-20.

Share, D. (1995). Phonological recoding and self-teaching: sine qua non of reading acquisition. Cognition, 55, 42-71.

Silva, C. y Alves, M. (2003). Relations between children's spelling and the development of phonological awareness. Educational Psychology, 23(1), 3-16.

Simmons, F. y Singleton, Ch. (2000). The reading comprehension abilities of dyslexic students in higher education. Dyslexia, 6, 178-192.

Snowling, M.J. (2001). From language to reading and dyslexia. Dyslexia, 7 37-46.

Shiel, G. (2002). Literacy Standard and factors affecting literacy: what national and international assessments tell us. En G. Reid y J. Wearmouth (eds.), Dyslexia and Literacy. Theory and Practice, 132-145. Chichester: John Wiley and Sons. 
Singleton, Ch. (2002). Dyslexia: cognitive factors and implications for literacy. En G Reid y J. Wearmouth (eds.), Dyslexia and Literacy. Theory and Practice, 115-129. Chichester: John Wiley and Sons.

Slocum, T.A., O’Connor, R.E. y Jenkins, J.R. (1993). Transfer among phonological manipulation skills. Journal of Educational Psychology, 85(4), 618-630.

Sodoro, J., Allinder, R.M. y Rankin-Erickson, J.L. (2002). Assessment of phonological awareness: review of methods and tools. Educational Psychology Review, !4(3), 223260.

Sofie, C.A. y Riccio, C.A. (2002). A comparison of multiple methods for the identification of children with reading disabilities. Journal of Learning Disablitiies, 35(3), 234-244.

Stahl, S.A. y Murray, B.A. (1994). Defining phonological awareness and its relationship to early reading. Journal of Educational Psychology, 86(2), 221-234.

Stein, J. (2001). The magnocellular theory of developmental dyslexia. Dyslexia, 7, 12-36.

Sterling, Ch. et al., (1998). Adult dyslexic writing. Dyslexia, 4, 1-15.

Stuart, K.M. (1986). Phonological awareness, letter-sound knowledge and learning to read. London: Doctoral Dissertation submitted to the University of London.

Swanson, H.L. y Deshker, D. (2003). Instructing adolescents with learning disabilities: converting a meta-analysis to practice. Journal of Learning Disabilities, 36(2), 124135.

Thomson, M.(1999). Subtypes of dyslexia: a teaching artefact?. Dyslexia, 5, 127-137.

Thomson, M. (2003). Monitoring dyslexics' intelligence and attainments: a follow-up study. Dyslexia, 9, 3-17.

Tonnessen, F.E. (1997). How can we best define Dyslexia?. Dyslexia, 3, 78-92.

Torgesen, J.K. y Wagner, R.K. (1999). Comprehensive tests of phonological processes Austin, TX: PRO-ED. 
Torgesen, J.K. et al., (1999). Preventing reading failure in young with phonological processing disabilities: group and individual responses to instruction. Journal of Educational Psychology, 91(4), 579-593.

Vellutino, F.R. y Scanlon, D.M. (2002). The interactive strategies approach to reading intervention. Contemporary Educational Psychology, 27, 573-635.

Vygotski, L.S. (1998) Los problemas fundamentales de la defectología contemporánea. Cultura y Educación, 11-12, 9-34.

Wearmouth, J. y Reid, G. (2002). Issues for assessment and planning of teching and learning. En G. Reid y J. Wearmouth (eds.), Dyslexia and Literacy. Theory and Practice, 151166. Chichester: John Wiley and Sons.

Weaver, C. (1990). Understanding whole language. Portsmouth, NH: Heineman.

Wright, J. y Jacobs, B. (2003). Teaching phonological awareness and metacognitive strategies to children with reading difficulties: a comparison of two instructional methods.

Educational Psychology, 23(1), 17-47.

Zabell, C. y Everatt, J. (2002). Surface and phonological subtypes of adult developmental dyslexia. Dyslexia, 8, 160-177. 